\title{
Integrated Solution for Anomalous Driving Detection Based on BeiDou/GPS/IMU Measurements
}

\author{
Rui $\mathrm{Sun}^{1}, \mathrm{Ke} \mathrm{Han}^{1,2}$, Jun $\mathrm{Hu}^{2}$, Yanjun Wang ${ }^{1}$, Minghua $\mathrm{Hu}^{1}$, Washington
}

Ochieng $^{1,2}$

${ }^{1}$ College of Civil Aviation, Nanjing University of Aeronautics and Astronautics, Nanjing 211100, China.

Email: rui.sun@nuaa.edu.cn

${ }^{2}$ Department of Civil and Environmental Engineering, Imperial College London, SW7 2AZ, United Kingdom.

\section{Abstract}

There has been an increasing role played by Global Navigation Satellite Systems (GNSS) in Intelligent Transportation System (ITS) applications in recent decades. In particular, centimetre/decimetre positioning accuracy is required for some safety related applications, such as lane control, collision avoidance, and intelligent speed assistance. Lane-level Anomalous driving detection underpins these safety-related ITS applications. The two major issues associated with such detection are (1) accessing high accuracy vehicle positioning and dynamic parameters; and (2) extraction of irregular driving patterns from such information. This paper introduces a new integrated framework for detecting lane-level anomalous driving, by combining Global Positioning Systems (GPS), BeiDou, and Inertial Measurement Unit (IMU) with advanced algorithms. Specifically, we use Unscented Particle Filter (UPF) to perform data fusion with different positioning sources. The detection of different types of Anomalous driving is achieved based on the application of a Fuzzy Inference System (FIS) with a newly introduced velocity-based indicator. The framework proposed in this paper yield significantly improved accuracy in terms of positioning and Anomalous driving detection compared to state-of-the-art, while offering an economically viable solution for performing these tasks.

Keywords: BeiDou, Anomalous Driving Detection, Unscented Particle Filter, Fuzzy Inference System 


$\begin{array}{ll}\text { DOP } & \text { Dilution of Precision } \\ \text { EKF } & \text { Extended Kalman Filter } \\ \text { EKFCA } & \text { Extended Kalman Filter with Constant Acceleration } \\ \text { EKFCTRA } & \text { Extended Kalman Filter with Constant Turn Rate and Acceleration } \\ \text { FIS } & \text { Fuzzy Inference System } \\ \text { FNN } & \text { Fuzzy Neural Network } \\ \text { GDOP } & \text { Geometric Dilution of Precision } \\ \text { GEO } & \text { Geosynchronous Orbit } \\ \text { GNSS } & \text { Global Navigation Satellite Systems } \\ \text { GPS } & \text { Global Positioning System } \\ \text { HDOP } & \text { Horizontal Dilution of Precision } \\ \text { IGSO } & \text { Inclined Geostationary Orbit } \\ \text { IMU } & \text { Inertial Measurement Unit } \\ \text { INS } & \text { Inertial Navigation System } \\ \text { ITS } & \text { Intelligent Transportation System } \\ \text { MEO } & \text { Medium Earth Orbit } \\ \text { PDOP } & \text { Position Dilution of Precision } \\ \text { PF } & \text { Particle Filter } \\ \text { UKF } & \text { Unscented Kalman Filter } \\ \text { UPF } & \text { Unscented Particle Filter } \\ \text { UPFCA } & \text { Unscented Particle Filter with Constant Acceleration } \\ \text { UPFCTRA } & \text { Unscented Particle Filter with Constant Turn Rate and Acceleration } \\ \text { VDOP } & \text { Vertical Dilution of Precision } \\ & \end{array}$

\section{Introduction}

The rapid growth of road transportation caused by urbanization and motorization has been accompanied by increased risk of traffic accidents. Reducing the occurrence of accidents requires not only the enhancement of the safety consciousness of the drivers and the general public, but also the development and deployment of technological measures to reduce transportation related risks. Intelligent Transportation System (ITS) technologies play a vital role in the assessment and improvement of driving safety, e.g. driving performance evaluation, anomalous driving detection, lane control, and collision avoidance. The safety performance of road transportation systems could be significantly enhanced when accurate and reliable information regarding anomalous driving detection is available and processed in a timely fashion. Real-time identification of vehicle driving patterns is a promising approach for anomalous driving detection. The underpinning idea is to extract anomalous driving cues from information obtained from the vehicle's motion sensors, including position, orientation, and velocity, to classify different dangerous driving styles and provide warning messages with recommended actions. 
Lecce et al. (2008) develop a driving information collection system based on a specific senor and GPS receiver, and apply pattern matching for the classification of driving styles. Their study is preliminary and does not present simulation or field test results. Chang et al. (2008) propose a machine learning mechanism based on Radial Basis Probability Neural Network (RBPNN) to calculate the safety level of a vehicle using trajectory and velocity data obtained from vision sensors. Both simulations and field tests are used to assess this method. However, this system only provides a coarse classification of the safety levels, namely "safe", "cautious", and "dangerous". In addition, the performance of the vision sensor is susceptible to adverse weather conditions during the field test. Similar methods have been developed by Imkamon et al. (2008) and Krajewski et al. (2009), with yet again crude classification of the hazard levels and no quantifiable performance indicators. Dai et al. (2010) combine mobile phones, accelerometers, and orientation sensors to detect vehicle manoeuvres typically associated with driving under influence. The acceleration patterns extracted from sensor readings are compared with patterns obtained from real-world driving tests. However, the success of such detection is not quantified, although it is argued that the system performance could be improved if GPS information were integrated. Saruwatari et al. (2012) introduce a method for detecting abnormal driving, which includes meandering, transverse motion, and sudden acceleration. The extraction of abnormal vehicle motions is performed by applying a multi-linear relationship in spatial-temporal images in the sense of group behaviour. This research, however, does not present any simulation or field experiment results.

Although the aforementioned real-time driving pattern detection approaches have shown some potential for anomalous driving detection, several technical barriers remain to be surmounted. Firstly, the effective use of vision sensors is largely dependent on weather conditions. Secondly, some methods heavily rely on the readings of high grade GPS or similar motion sensors, the cost of which may hinder their widespread practical use. Moreover, most of the anomalous driving detection systems discussed before are still at an early stage of development, with few simulations or field tests. Last but no least, most such systems do not offer a robust algorithm to distinguish or quantify different types of anomalous driving styles, and their efficiency and reliability need to be further examined.

In order to address these issues, Sun et al. (2015) propose an integrated solution for lane-level anomalous driving detection, which takes advantage of high accuracy estimation of vehicle positions and dynamic parameters, and combines them with different types of anomalous driving identification method. That paper has identified a critical threshold $(0.5 \mathrm{~m})$ in terms 
of the positioning accuracy, where only positioning errors below this value ensure the validity and effectiveness of the lane-level anomalous driving detection. Unfortunately, due to the insufficient accuracy of GPS positioning, it is impossible to achieve an accuracy of $0.5 \mathrm{~m}$ for all instances, and the capability of any positioning mehtods to reach such accuracy is of critical importance to the successful detection of anomalous driving. In this paper, we have made considerable improvement over the method by Sun et al. (2015) in terms of accuracy, reliability, and practicality. This is done through both technological innovation and algorithm advancement. In particular, we introduce BeiDou as a new source of positioning data, and propose a new UPF-based GNSS/IMU fusion model.

BeiDou is a Chinese satellite navigation system initiated in 2000. By the end of 2012, the basic system construction was finished, and it has, since then, covered China and AsiaPacific areas by providing regional continuous positioning, navigation, timing and short message communication services. It is expected that the BeiDou system will reach a global coverage and become one of the four largest Global Navigation Satellite Systems (GNSSs) in the world by 2020, along with GPS, GLONASS and Galileo (Yang, 2010). Currently, 20 BeiDou satellites have been launched, among which 14 are in operation, including 5 Geosynchronous Orbit (GEO) satellites, 5 Inclined Geostationary Orbit (IGSO) satellites and 4 Medium Earth Orbit (MEO) satellites (Sun et al., 2012). Not only can the BeiDou system be used independently for single-mode positioning, it can also be combined with GPS and other positioning systems to fix positioning solutions (Xiao et al., 2014; Cai et al., 2014; Odolinski et al., 2014a, 2014b). For the open regional service of BeiDou, it is claimed that its open application accuracy is close to that of GPS (Chen et al., 2009; Xu et al., 2013). Combining BeiDou with GPS for positioning purposes brings more satellites redundancies and thus has great potential to improve the positioning accuracy, which is beneficial not only for the anomalous driving detection as we study in this paper, but also a lot of other ITS applications.

This paper proposes a lane-level anomalous driving detection framework, and makes contribution in the following three areas:

1. Introducing BeiDou as an additional source of positioning;

2. Two new methods for the fusion of positioning data and motion parameter estimation; and

3. A new anomalous driving detection algorithm based on Fuzzy Inference System and velocity-based indicator. 
The significance of each contribution is illustrated below.

The benefit of having both GPS and BeiDou signals is illustrated in detail in this paper. In particular, it is shown that based on a combination of both GPS and BeiDou sources, the positioning accuracy is greatly enhanced, regardless of the data fusion model employed (see Table 7). Moreover, the inclusion of BeiDou does not bring additional economic cost since cheap receiver chips can be manufactured to pick up both GPS and BeiDou signals. Furthermore, using the newly introduced BeiDou source, we further devise data fusion algorithms based on Unscented Kalman Filter (UKF) or Unscented Particle Filter (UPF), which provides substantial improvements over Extended Kalman Filter (EKF) and Particle Filter (PF) considered in Sun et al. (2015); see Table 1 for more detailed comparison.

Regarding the identification of anomalous driving patterns, we consider a velocity-related indicator and incorporate it into the Fuzzy Interface System (FIS), which has not been done in the literature. The new anomalous driving detection algorithm is extensively tested and compared with other state-of-the-art algorithms, including those from Sun et al. (2015), Chang et al. (2008), Dai et al. (2010), and Krajewski et al. (2009). Our experiments based on both simulated and field data show that the proposed FIS algorithm outperforms, by a discernible margin, all other methods in terms of timeliness of detection, availability, and correction rate. In particular, given the same positioning data input, our method is $0.1 \mathrm{~s}-4 \mathrm{~s}$ earlier than the other methods in terms of time of first detection (see Table 8). Moreover, it also provides higher availability and correction rate ${ }^{1}$ across all scenarios than the state-of-thearts, with a margin of $0.7 \%-3 \%$ (availability) and $0.16 \% \sim 17 \%$ (correction rate); see Table 9.

Finally, the complete, end-to-end anomalous driving detection framework, including data acquisition, fusion, and irregularity detection, is tested using field data and GPS/BeiDou signals. Due to the improvements made at individual stages of the framework as we briefly described above, the overall performance of the framework yields a significant improvement over our previous work (Sun et al., 2015). The use of GPS, BeiDou and commercial IMU as data sources for lane-level anomalous driving detection has not been done before to the best of our knowledge. The field test has demonstrated the practicality of our technical approach. Without the need for vision or other high-grade sensors, the anomalous driving detection method proposed here significantly reduces the cost, and becomes an economically viable

\footnotetext{
${ }^{1}$ Availability refers to the percentage of valid driving style identification (weaving, swerving, jerky driving, and normal driving) produced by the algorithm. Correct detection rate is the percentage of correct driving style identification among all available outputs.
} 
solution. The improvements we have made over other state-of-the-art algorithms or frameworks are of paramount importance to driving safety and accident prevention.

The rest of the paper is organized as follows. Section 2 presents the BeiDou/GPS and IMU fusion models for the lane-level positioning and dynamic parameter estimation. Several data fusion models are proposed and compared as well. Section 3 presents the FIS-based anomalous driving detection algorithm and its advantage over existing ones by taking into account the velocity indicator. In Section 4 we conduct a field test, in which the improvement of individual component of the proposed anomalous driving detection framework, including BeiDou data source, UKP/UPF data fusion models, and FIS-based anomalous driving detection, is analysed in detail using real-world data. We also test the complete, end-to-end anomalous driving detection framework with all the new components in place, and illustrate its advantage over state-of-the-art. Finally, Section 5 provides some concluding remarks.

\section{Sensor Integration Model}

This section describes a sensor integration model for lane-level positioning based on:

(1) Data fusion methods using Unscented Kalman Filter (UKF) and Unscented Particle Filter (UPF); and

(2) Precise vehicle motion models for straight and curved lanes.

Sun et al. (2015) employ Extended Kalman Filter (EKF) and Particle Filter (PF) to estimate position and motion parameters. It is shown that PF yields better results than EKF. Nevertheless, in some cases, PF could produce large estimation errors when the likelihood of the input measurements is too peaked or the noise of the measurements is heavy-tailed, which are consequences of inaccurate sensors or sudden changes of the input data (Van der Merwe et al., 2000). Recently some more advanced filters, namely those based on generic PF or EKF, have emerged for non-linear estimations. Examples include Unscented Kalman Filter and Unscented Particle Filter, which are potentially beneficial to high-precision positioning and parameter estimation. Sections 2.1 and 2.2 below describe the UKF and UPF methods in detail for the intended application, while Section 2.3 compares the estimation results for different fusion models.

\subsection{Unscented Kalman Filter Model Design}

For nonlinear systems, UKF treats the nonlinear state vector without calculating the Jacobian, 
which is the essential linearization step in the EKF method. Instead, UKF employs the socalled Unscented Transformation (UT) for calculating the statistics of random variables in the process model. In UKF, the state approximated by a Gaussian Random Variable (GRV) is represented by a set of carefully chosen points, namely sigma points. The true mean and covariance of the GRV are captured by these sigma points, which will also capture the posterior mean and covariance accurately up to the second order for any nonlinearity propagating through the system (Wan and Van Der Merwe, 2000). The main steps for the UKF have been tailored to fit the specific application and are presented below.

\section{UKF Algorithm:}

$\chi_{\mathrm{k}} \quad$ Sigma vector at step $\mathrm{k}$

$\hat{\mathrm{X}}_{\mathrm{k}} \quad$ estimated state vector at step $\mathrm{k}$

$\hat{y}_{\mathrm{k}} \quad$ estimated measurement vector at step $\mathrm{k}$

$\mathrm{y}_{\mathrm{k}} \quad$ measurement vector at step $\mathrm{k}$

$r \quad$ scaling parameter

$\mathrm{P}_{\mathrm{k}}^{\mathrm{XX}} \quad$ covariance matrix of the state vector at step $\mathrm{k}$

$\mathrm{P}_{\mathrm{k}}^{\mathrm{YY}} \quad$ covariance matrix of the measurement vector at step $\mathrm{k}$

$\mathrm{P}_{\mathrm{k}}^{\mathrm{XY}} \quad$ cross covariance matrix of the state vector and measurement vector at step $\mathrm{k}$

$\mathrm{W}_{\mathrm{i}}^{(\mathrm{m})} \quad$ weights for sample mean with $\mathrm{i}$-th column of the matrix

$\mathrm{W}_{\mathrm{i}}^{(\mathrm{C})} \quad$ weights for covariance with $\mathrm{i}$-th column of the matrix

$\mathrm{Q}_{\mathrm{k}} \quad$ covariance matrix of process noise at step $\mathrm{k}$

$\mathrm{R}_{\mathrm{k}} \quad$ covariance matrix of measurement noise at step $\mathrm{k}$

1) Initialization. The initialization is carried out for the estimated state vector $\hat{x}_{0}$ and covariance matrix $P_{0}^{X X}$ on the defined state vector for the lane-level anomalous driving. The state vector is defined as

where:

$$
(\mathrm{XYv} \beta \theta \omega)^{T}
$$

$\mathrm{X}$ is the $\mathrm{X}$-coordinate (in meter) of the vehicle's geometric center in the local coordinate system;

$\mathrm{Y}$ is the Y-coordinate (in meter) of the vehicle's geometric center in the local coordinate system;

$\mathrm{v}$ is the velocity along the heading direction;

$\beta$ is the angle between the tangent line of the lane central line and the $\mathrm{x}$-axis;

$\theta$ is the angle between the vehicle heading and the $\mathrm{x}$-axis;

$\omega$ is the vehicle yaw rate.

2) Calculate the Sigma points. For $k \geq 1$,

$$
\chi_{k-1}=\left[\begin{array}{lll}
\hat{x}_{k-1} & \hat{x}_{k-1}+r \sqrt{P_{k-1}^{X X}} & \hat{x}_{k-1}-r \sqrt{P_{k-1}^{X X}}
\end{array}\right]
$$

where the three column vectors form a matrix.

3) Propagate the sigma points. Using the state-update function $\chi_{k \mid k-1}=F\left(\chi_{k-1}, u_{k-1}\right)$ to transform the sigma points and then calculate the a priori state estimate using (3) and 
covariance using (4). The function $F(\cdot, \cdot)$ is related to the model of the vehicle's dynamic (Gyorgy et al., 2014).

$$
\begin{gathered}
\hat{x}_{k \mid k-1}=\sum_{i=0}^{2 L} W_{i}^{(m)} \chi_{k \mid k-1}^{i} \\
P_{k}^{X X}=\sum_{i=0}^{2 L} W_{i}^{(C)}\left[\chi_{k \mid k-1}^{i}-\hat{x}_{k \mid k-1}\right]\left[\chi_{k \mid k-1}^{i}-\hat{x}_{k \mid k-1}\right]^{T}+Q_{k}
\end{gathered}
$$

where $\chi_{k \mid k-1}^{i}$ denotes the i-th column of the matrix $\chi_{\kappa \mid \kappa-1}$.

4) Update the output vectors. Transform the sigma points through the measurement-update function $h(\cdot)$ (Gyorgy et al., 2014), and calculate the mean and covariance of the measurement vector according to (5) and (6) respectively.

$$
\begin{gathered}
\hat{y}_{k \mid k-1}=\sum_{i=1}^{2 L} W_{i}^{(m)} h\left(\chi_{k \mid k-1}^{i}\right) \\
P_{k}^{Y Y}=\sum_{i=0}^{2 L} W_{i}^{(C)}\left[h\left(\chi_{k \mid k-1}^{i}\right)-\hat{y}_{k \mid k-1}\right]\left[h\left(\chi_{k \mid k-1}^{i}\right)-\hat{y}_{k \mid k-1}\right]^{T}+R_{k}
\end{gathered}
$$

5) Calculate the cross covariance matrix and the Kalman Filter gain. Calculate the cross covariance matrix and the Kalman Filter gain vector according to (7) and (8), respectively:

$$
\begin{gathered}
P_{k}^{X Y}=\sum_{i=0}^{2 L} W_{i}^{(C)}\left[\chi_{k \mid k-1}^{i}-\hat{x}_{k \mid k-1}\right]\left[h\left(\chi_{k \mid k-1}^{i}\right)-\hat{y}_{k \mid k-1}\right]^{T} \\
K_{k}=P_{k}^{X Y}\left(P_{k}^{Y Y}-R_{k}\right)^{-1}
\end{gathered}
$$

6) Calculate the estimated state and the covariance. Calculate the estimated state and the covariance in accordance with the generic Kalman Filter.

$$
\begin{gathered}
\hat{x}_{k \mid k}=\hat{x}_{k \mid k-1}+K_{k}\left(y_{k}-\hat{y}_{k \mid k-1}\right) \\
P_{k \mid k}=P_{k \mid k-1}-K_{k} P_{k}^{Y Y} K_{k}^{T}
\end{gathered}
$$

\subsection{Unscented Particle Filter Model Design}

The Unscented Particle Filter (UPF) is a combination of the unscented method and Particle Filters. As is known from previous research (Wan and Van Der Merwe, 2000), the insufficiency of valid particles is an issue for generic PF, and is caused by using the transition prior as proposal distribution. However, if the unscented filter is used as proposal distribution, the particles will be moved towards the regions of high likelihood. This can be simply achieved by propagating the sufficient statistics of the UKF for each particle (Van Der Merwe and Wan, 2000). The following steps describe the UPF cycle for lane-level positioning, where the notations have the same meaning as in the previous section.

\section{UPF Algorithm:}


1) Initialization. Define the state vector as:

$$
(\mathrm{X} Y \mathrm{v} \theta \omega \mathrm{a} \beta \mathrm{d})^{T}
$$

where a is the vehicle acceleration along the heading; $d$ is the vehicle lateral displacement. Two sub-state vectors, vehicle motion vector (12) and lane geometry-related vector (13), are derived from the state vector (11). Sub-state vector (12) is for the UPF cycle calculation and (13) is the dependent vector of (12).

$$
\begin{gathered}
p(k)=(\mathrm{X} Y \mathrm{v} \theta \omega \mathrm{a})^{T} \\
q(k)=(\beta d)^{T}
\end{gathered}
$$

In the UPF, the state variables change with time (indicated using $k$ ), and they are associated with each particle (indicated using $i$ ). The detail of initialization of the particles for $p(k)$ and $q(k)$ is the same as the generic PF discussed in Sun et al. (2015).

2) Filter Prediction. The prediction model for $p(k)$ is based on the vehicle motion models. In this paper, constant acceleration (CA) models are applied to straight highway motion, while Constant Turn Rate and Acceleration (CTRA) models are applied to curved scenarios (Tsogas et al., 2005; Sun et al., 2015). The quantity $q(k)$ is predicted based on the geometric relationship with the lane segment as follows:

$$
\begin{gathered}
\beta_{k+1}^{i} \approx \beta_{k}^{i} \\
d_{k+1}^{i}=d_{k}^{i}+\sin \left(\beta_{k}^{i}\right) \Delta_{x}^{i}-\cos \left(\beta_{k}^{i}\right) \Delta_{y}^{i}
\end{gathered}
$$

where $\Delta_{x}^{i}$ and $\Delta_{y}^{i}$ indicate the displacement along the $\mathrm{x}$ - and y-axis, respectively.

3) Filter update. The prediction cycle is applied to every input sample. First, the validity of $d^{i}$ is checked. Once this is done, we apply the UKF to update the particles. The covariance is updated according to (10) and the state estimate is updated based on (9).

4) Normalize and resample. After every update phase, the particles' weights are modified, and the normalization and resample test phases of a UPF is launched.

\subsection{Comparison of Model Performances}

Different types of driving styles are defined in straight and curved lanes on highways. The driving styles can be classified into weaving, swerving, jerky driving and normal driving 
(NHTSA, 2010; Sun et al., 2015). In this paper, these same driving styles are considered for the anomalous driving detection.

The proposed position and motion estimation methods (UKF and UPF) are tested using simulation data here, before they are examined with field tests (see Section 4). The simulation data generated by the GNSS simulator and Matlab are used to feed the UKF and UPF for the evaluation of the positioning results. Details of the data generation process are described in Sun et al. (2015) and omitted here. Two motion models are used in this paper: Constant Acceleration (CA) and Constant Turn Rate and Acceleration (CTRA), which have the best performance when combined with sensor fusion methods (Sun et al., 2015). Consequently, for straight lanes the proposed fusion models are referred to as Unscented Kalman Filter with Constant Acceleration (UKFCA) and Unscented Particle Filter with Constant Acceleration (UPFCA); for the curved lane the new fusion models are called Unscented Kalman Filter with Constant Turn Rate and Acceleration (UKFCTRA) and Unscented Particle Filter with Constant Turn Rate and Acceleration (UPFCTRA).

Comparison of the proposed fusion methods with those proposed by Sun et al. (2015) (i.e. KF and PF with CA and CTRA) is summarized in Table 1, where the 2d root-mean-square (2dRMS) is used as the criterion. It is shown that the UKFCA/UKFCTRA and UPFCA/UPFCTRA methods have better performance than EKFCA/EKFCTRA and PFCA/PFCTRA. Especially, the UPFCA/UPFCTRA yields the highest positioning accuracy among all others in their designed scenarios. It is also noted from the table that the 2dRMS accuracy for S4 (Normal Driving on straight) are lower than the other straight scenarios for all four methods. The likely reason is that for normal driving on straight scenarios the motion is almost linear; however, EKF, PF, UKF, UPF are all nonlinear filters, which may incur larger errors than nonlinear scenarios.

Table $12 \mathrm{dRMS}$ accuracy for straight and curved scenarios.

\begin{tabular}{|l|c|c|c|c|}
\hline \multicolumn{5}{|c|}{ Straight Scenarios $(\mathrm{m})$} \\
\hline Anomalous driving scenarios & UKFCA & UPFCA & EKFCA & PFCA \\
\hline S1 Weaving on straight & 0.2034 & 0.1521 & 0.3621 & 0.2142 \\
\hline S2 Swerving on straight & 0.3090 & 0.2560 & 0.5653 & 0.3410 \\
\hline S3 Jerky Driving on straight & 0.3238 & 0.2875 & 0.4801 & 0.3713 \\
\hline S4 Normal Driving on straight & 0.3654 & 0.3149 & 0.4920 & 0.4215 \\
\hline \multicolumn{7}{|l|}{ Curved Scenarios (m) } \\
\hline Anomalous driving scenarios & UKFCTRA & UPFCTRA & EKFCTRA & PFCTRA \\
\hline S5 Weaving on curve & 0.3546 & 0.2942 & 0.5003 & 0.3906 \\
\hline S6 Swerving on curve & 0.3216 & 0.2843 & 0.4502 & 0.3735 \\
\hline S7 Jerky Driving on curve & 0.3761 & 0.3204 & 0.6337 & 0.4315 \\
\hline S8 Normal Driving on curve & 0.2917 & 0.2381 & 0.3924 & 0.3532 \\
\hline
\end{tabular}




\section{Anomalous Driving Detection}

The detection of a vehicle's anomalous driving styles is by matching the estimated driving styles with reference styles. In this paper, besides the yaw rate and lateral displacement, which are considered in Sun et al. (2015), the vehicle's velocity is added as the third input to improve the representation of the vehicle's motion. We denote the vehicle's yaw rate, lateral displacement, and velocity as omega, $d$ and $v$, respectively. In order to reduce noises within the quantities obtained from the filters and extract trends of their changes, Moving Average Deviation (MAD) is applied to omega, $d$ and $v$, and the results are respectively denoted as Oindicator, D-indicator and $\mathrm{V}$-indicator. The detection of anomalous driving has to recognize a driving event, which characterizes the different driving styles based on Fuzzy Inference System (FIS), with O-indicator and D-indicator V-indicator derived at every time epoch.

\subsection{System Overview}

The flow chart in Figure 1 illustrates the overall lane-level anomalous driving detection system proposed in this paper. The system consists of two phases. The first phase is concerned with lane-level positioning and motion parameter estimation, as we have described in Section 2. For the collection of the vehicle's driving data, we mount one Inertial Measurement Unit (IMU) with one gyro and one accelerometer along the vehicle body axis to output the yaw rate, acceleration and heading angle. Meanwhile, a GPS/BeiDou receiver is used to collect the vehicle's local coordinates and heading velocity. These data are then provided to the fusion model (Filter Prediction) to estimate the position and motion parameters of the vehicle ( $v$, omega, and $d)$.

The second phase involves the anomalous driving detection with input (position and motion parameters) from the first phase. Specifically, omega, $d$, and $v$ from the first phase are used to calculate the O-indicator, D-indicator and V-indicator. Then, these three indicators are used as the inputs of the FIS for producing the risk type indicator, based on which the driving classification indicator is developed in order to identify the main features of each driving style. Finally, by comparing sorted values of the driving classification indicators with predefined sorting rules extracted from the experience data ${ }^{2}$, one can obtain the type of driving style.

\footnotetext{
${ }^{2}$ The experience data are provided by the Zhoushan Traffic Police Station; the fuzzy values and rules are obtained as the outputs of artificial neural networks trained based on the recent 5-year vehicle trajectory data in radar image format.
} 


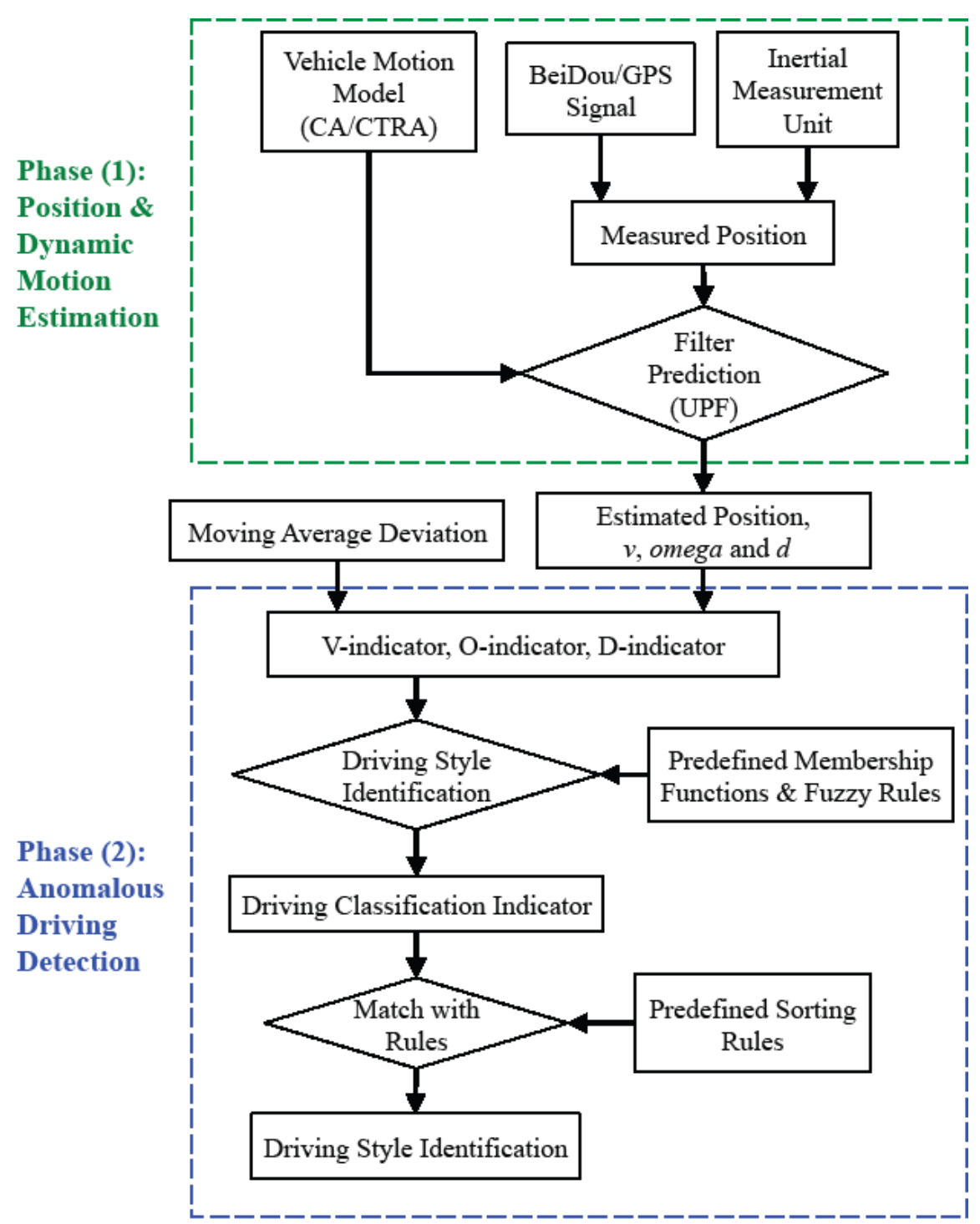

Figure 1 Framework of the proposed anomalous driving detection system.

\subsection{FIS-Based Anomalous Driving Detection Algorithm with Velocity Indicators}

The O-indicator, D-indicator and V-indicator are the inputs of the FIS system for calculating the risk type indicator. The membership function and fuzzy rules for the input indicators and output indicator are designed in this section. Figure 2 shows an example of the designed FIS membership functions for the O-indicator, D-indicator, $\mathrm{V}$-indicator and risk type indicator in straight lane scenarios. The fuzzy values of these indicators are defined in Table 2. The risk type indicator is defined with four fuzzy values A, B, C, D, representing low risk, medium risk, high risk, and very high risk, respectively (see Figure 2). 
Table 2 Acronyms for different fuzzy values of the indicators.

\begin{tabular}{|l|l|l|l|l|}
\hline & Small & Medium & Large & Very large \\
\hline V-indicator & SV & MV & LV & VLV \\
\hline O-indicator & SO & MO & LO & VLO \\
\hline D-indicator & SD & MD & LD & VLD \\
\hline
\end{tabular}
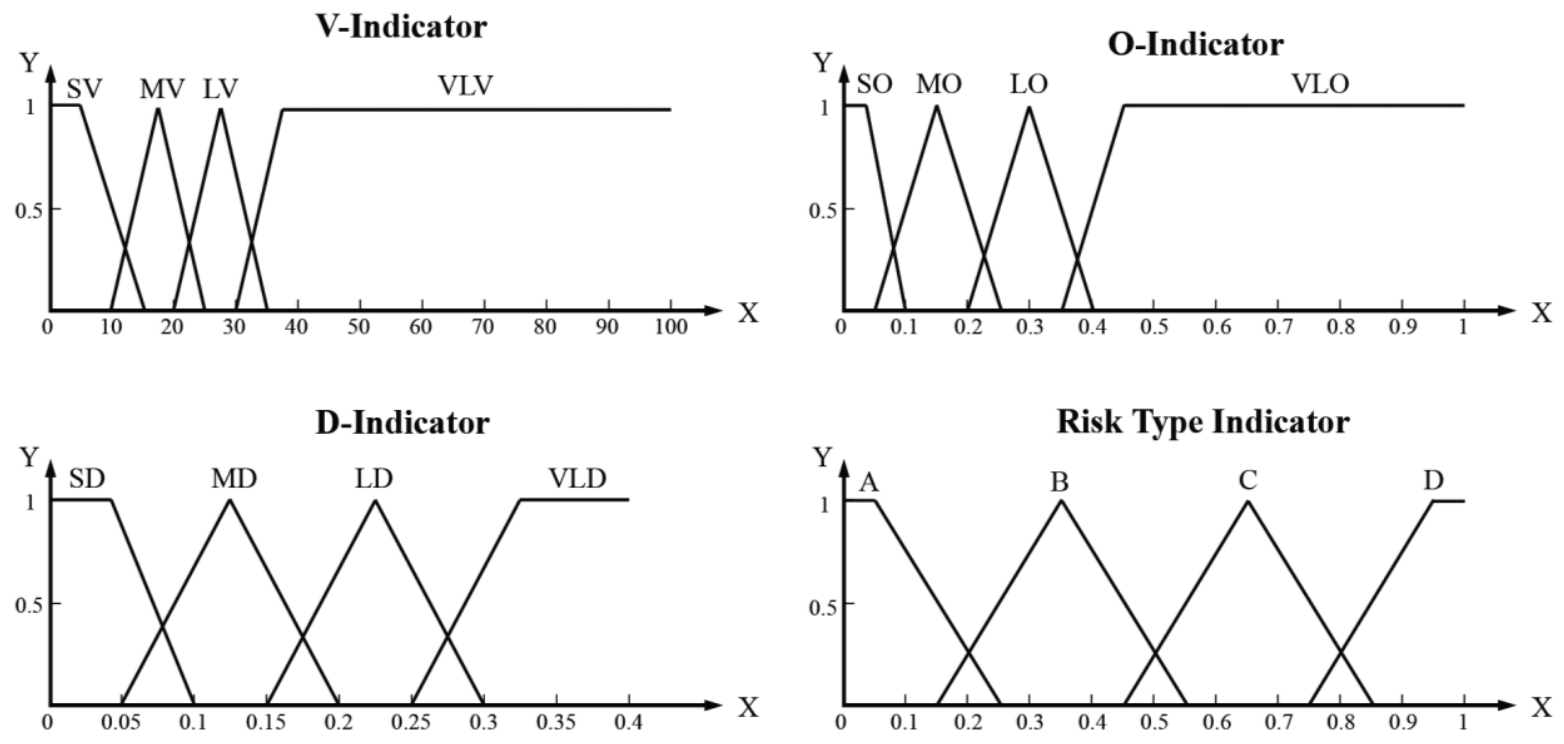

Figure 2 Membership function for the straight scenarios.

The experience data are used to design the fuzzy values in the membership functions and the rules for mapping the $\mathrm{V}$-indicator, O-indicator and $\mathrm{D}$-indicator to the corresponding risk types. The experience data are provided by the Zhoushan Traffic Police Station; the fuzzy values and rules are obtained as the outputs of artificial neural networks trained based on the recent 5-year vehicle trajectory data in radar image format. The mapping rules for the straight-lane scenarios are shown in Table 3, while the rules for the curved-lane scenarios are similar and omitted here.

Table 3 Rules of FIS for the straight scenarios.

\begin{tabular}{|l|l|l|l|}
\hline O-indicator & D-indicator & V-indicator & Risk Type \\
\hline SO & SD & Any(SV,MV) & A \\
\hline SO & SD & LV & B \\
\hline SO & MD & SV & B \\
\hline SO & SD & VLV & C \\
\hline SO & Any(MD,LD) & MV & C \\
\hline SO & Any(LD,VLD) & SV & C \\
\hline SO & Any(MD,LD,VLD) & Any(LV,VLV $)$ & D \\
\hline MO & Any( SD,MD,LD,VLD) & Any(LV,VLV) & D \\
\hline MO & Any(MD,LD,VLD) & Any(SV,MV) & C \\
\hline LO & Any(SD,MD,LD,VLD) & Any(SV,MV,LV,VLV) & D \\
\hline VLO & Any(SD,MD,LD,VLD) & Any(SV,MV,LV,VLV) & D \\
\hline
\end{tabular}


Based on the risk type indicator produced by the FIS in every time epoch, the counts of all four risk types (A, B, C, D) for all the driving scenarios (defined in Table 1) during the last 5 seconds are recorded. Four driving classification indicators are then developed to classify different types of anomalous driving. They are $\mathrm{AB}, \mathrm{BC}, \mathrm{CD}$, and $\mathrm{AD}$; and their values are calculated based on the counts of relevant risk types: $A B$ is defined to be the sum of counts of risk type $\mathrm{A}$ and risk type $\mathrm{B}$; the others can be similarly defined.

Once the numerical values of the driving classification indicators are calculated, one can sort these indicators and use the sorted list to identify the types of anomalous driving based on the experience data. For example, if the order $A B>B C>=C D>=A D$ is associated with weaving in the experience data, then the driving style is classified as weaving whenever the same sorting pattern is identified from the test data. Notice that there may be more than one sorting patterns associated with the same anomalous driving style. We refer the reader to Sun et al. (2015) for more elaboration of the development of the driving classification indicators and the sorting rules.

\subsection{Algorithm Test based on Simulated Data}

In this section we conduct a preliminary assessment of the proposed anomalous driving detection algorithm based on simulated data, and compare it with the algorithm proposed in Sun et al. (2015), which does not take V-indicator into account. An episode of simulated driving data is generated to find out if the newly designed algorithm can continuously detect different types of anomalous driving. A time horizon of $120 \mathrm{~s}$ is assumed, and the simulated data include manoeuvres corresponding to weaving for 20 seconds, swerving for 20 seconds, jerky driving for 20 seconds and normal driving for 60 seconds. The anomalous driving detection results are generated at three different frequencies: $10 \mathrm{~Hz}, 5 \mathrm{~Hz}$ and $1 \mathrm{~Hz}$; see Table 4 for a summary of the results. Here, we use the first time of correct detection as the performance measure.

Table 4 Time to first detection in the simulation test.

\begin{tabular}{|c|c|c|c|c|}
\hline \multirow{2}{*}{$\begin{array}{c}\text { Output } \\
\text { Frequency }\end{array}$} & \multicolumn{4}{|c|}{ FIS Algorithm with (V-indi, O-indi and D-indi) input } \\
\cline { 2 - 5 } & Weaving & Swerving & Jerky Driving & Normal Driving \\
\hline $10 \mathrm{~Hz}$ & $5 \mathrm{~s}$ & $24.3 \mathrm{~s}$ & $41.6 \mathrm{~s}$ & $62.5 \mathrm{~s}$ \\
\hline $5 \mathrm{~Hz}$ & $5 \mathrm{~s}$ & $24.5 \mathrm{~s}$ & $42 \mathrm{~s}$ & $62.5 \mathrm{~s}$ \\
\hline $1 \mathrm{~Hz}$ & $5 \mathrm{~s}$ & $25 \mathrm{~s}$ & $42 \mathrm{~s}$ & $63 \mathrm{~s}$ \\
\hline \multirow{2}{*}{$\begin{array}{c}\text { Output } \\
\text { Frequency }\end{array}$} & \multicolumn{4}{|c|}{ FIS Algorithm with (O-indi and D-indi) input } \\
\cline { 2 - 5 } & Weaving & Swerving & Jerky Driving & Normal Driving \\
\hline $10 \mathrm{~Hz}$ & $5 \mathrm{~s}$ & $24.5 \mathrm{~s}$ & $41.8 \mathrm{~s}$ & $62.7 \mathrm{~s}$ \\
\hline $5 \mathrm{~Hz}$ & $5 \mathrm{~s}$ & $24.5 \mathrm{~s}$ & $42 \mathrm{~s}$ & $63 \mathrm{~s}$ \\
\hline $1 \mathrm{~Hz}$ & $5 \mathrm{~s}$ & $25 \mathrm{~s}$ & $42 \mathrm{~s}$ & $63 \mathrm{~s}$ \\
\hline
\end{tabular}


Table 4 suggests that the newly designed FIS algorithm for anomalous driving detection, with the additional consideration of the $\mathrm{V}$-indicator, has slightly improved the time to first detection. The savings are typically between $0.2-0.5 \mathrm{~s}$, and the improvement is more significant for high-frequency outputs.

Availability and correct detection rate are two other important performance indicators for evaluating the effectiveness of the anomalous driving detection algorithms. Availability refers to the percentage of valid driving style identification (weaving, swerving, jerky driving, and normal driving) produced by the algorithm. Correct detection rate is the percentage of correct driving style identification among all available outputs. The availability and correct detection rate for the simulation test is shown in Table 5, which clearly indicates that the proposed algorithm outperforms the one without the $\mathrm{V}$-indicator in terms of both criteria.

Table 5 Availability and correct detection rate in the simulation test.

\begin{tabular}{|c|c|c|}
\hline \multicolumn{2}{|c|}{ FIS Algorithm with (V-indi, O-indi and D-indi) input } \\
\hline Output Frequency & Availability & Correct Detection Rate \\
\hline $10 \mathrm{~Hz}$ & $95.14 \%$ & $98.10 \%$ \\
\hline $5 \mathrm{~Hz}$ & $97.28 \%$ & $99.29 \%$ \\
\hline $1 \mathrm{~Hz}$ & $98.35 \%$ & $100 \%$ \\
\hline \multicolumn{2}{|c|}{ FIS Algorithm with (O-indi and D-indi) input } \\
\hline Output Frequency & Availability & Correct Detection Rate \\
\hline $10 \mathrm{~Hz}$ & $94.08 \%$ & $97.12 \%$ \\
\hline $5 \mathrm{~Hz}$ & $97.16 \%$ & $98.15 \%$ \\
\hline $1 \mathrm{~Hz}$ & $98.20 \%$ & $100 \%$ \\
\hline
\end{tabular}

So far, we have discussed two new data fusion methods, UKFCA/UKFCTRA and UPFCA/UPFCTRA for positioning and motion parameter estimation (Section 2). The outputs of these methods are then input into the anomalous driving detection algorithm proposed in this section. The efficacy of the IFS-based anomalous driving detection with V-indicator is proven with simulated data. In the next section, the entire end-to-end anomalous driving detection framework, including BeiDou/GPS+IMU data acquisition, data fusion, and anomalous driving detection, will be tested extensively using real data from a field test.

\section{Field Experiment and Analysis}

A field test was carried out to validate the proposed lane-level anomalous driving detection approach based on real BeiDou/GPS \& IMU data filtered with the proposed UPFCA and UPFCTRA methods (the UPF method is selected over the UKF since it has better performance as shown in Table 1). Since this paper makes contribution in both data fusion 
and anomalousity detection (see Figure 1), the test is designed into three stages: (1) to validate the proposed lane-level high-precision positioning approach, i.e. UPFCA and UPFCTRA fusion methods based on BeiDou/GPS and IMU feeds (to be conducted in Section 4.2); (2) to validate the proposed new FIS-based anomalous driving detection algorithm and compare it with other state-of-the-art algorithms for the same application, based on the same reference data ${ }^{3}$ (to be conducted in Section 4.3); and (3) to test the integrated GPS/BeiDoufusion-detection approach (to be conducted in Section 4.4).

\subsection{Data Collection}

The testing field is on the Ningbo-Zhoushan expressway near the University of Nottingham, Ningbo campus. The driving data were captured for 2 hours from 13:50 to 15:50, which involved weaving, swerving and jerky driving on both straight and curved lanes. A total of six sessions, involving different types of anomalous driving styles, are defined in Table 6. All the sessions were carried out on open areas, when there was no passing-by traffic or roadside objects to ensure the safety of the experimental environment and also the high quality of the GPS and BeiDou measurements.

Table 6 Definition of sessions.

\begin{tabular}{|c|c|c|c|}
\hline Session Name & Start Time (UTC) & End Time (UTC) & Driving Type \\
\hline Session 1 & $13: 51: 24.0$ & $13: 51: 31.2$ & Weaving on Straight \\
\hline Session 2 & $13: 54: 25.5$ & $13: 55: 01.4$ & Jerky Driving on Straight \\
\hline Session 3 & $14: 00: 10.0$ & $14: 00: 17.5$ & Swerving on Straight \\
\hline Session 4 & $14: 57: 13.9$ & $14: 57: 23.5$ & Jerky Driving on Curve \\
\hline Session 5 & $15: 17: 05.8$ & $15: 17: 11.6$ & Swerving on Curve \\
\hline Session 6 & $15: 21: 25.7$ & $15: 21: 35.2$ & Weaving on Curve \\
\hline
\end{tabular}

For the anomalous driving data collection, the test vehicle was driven at speeds ranging from $70 \mathrm{~km} / \mathrm{h}$ to $120 \mathrm{~km} / \mathrm{h}$, and the positioning data were collected at a frequency of $10 \mathrm{~Hz}$. The installation of various equipment used in the experiment is illustrated in Figure 3, The equipment specification are as follows.

- JAVARD Triumph-VS receiver for real-time GNSS data collection, including the BeiDou and GPS positioning results and velocity of the vehicle.

- XSENS IMU for real-time attitude data collection, including heading angle and yaw rate of the vehicle.

\footnotetext{
${ }^{3}$ The reference data are obtained using high-accuracy I-Mar RT-200 INS outputs, which were postprocessed by forward and backward processing tools to yield highly accurate vehicle positioning information, which were treated as ground truth.
} 
- i-Mar RT-200 INS for providing integrated GPS/INS measurements, which were post-processed by forward and backward processing tools for a high accuracy reference. The reference data are treated as the ground truth.

Besides the collection of car trajectory data, another important quantity to measure is the lane's central line in the driving route. In this experiment, the coordinates of the lane's central line are collected afterwards by the vehicle with i-Mar RT-200 INS (with 20Hz output rate) driving along the middle of the same lane on which the anomalous driving were conducted. The post-processed i-Mar RT-200 measurements are recognized as the position of the lane's central line. The lateral displacement of the vehicle was calculated by finding the two measurement points on the central line that are closest to the vehicle, and then calculating the perpendicular distance from the vehicle to the line segment containing these two points.

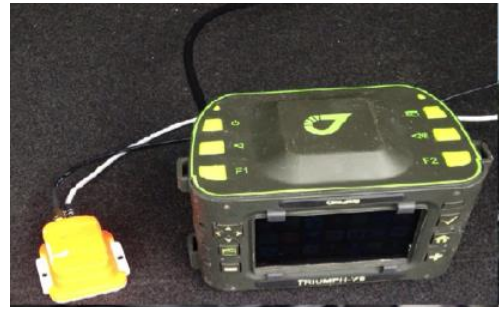

GNSS receiver integrated with IMU

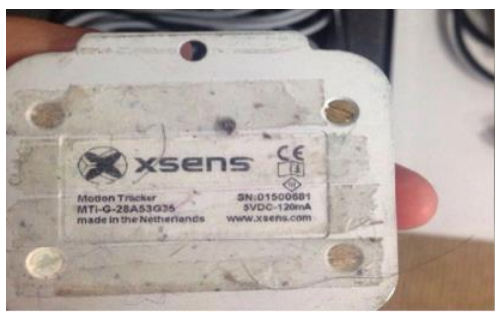

XSENS IMU

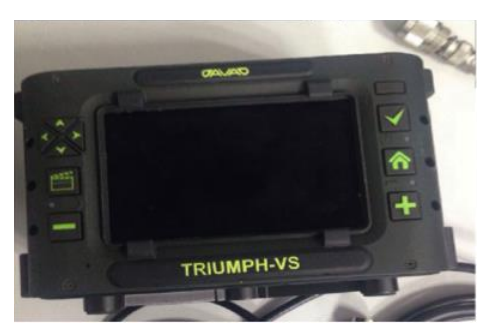

GNSS receiver

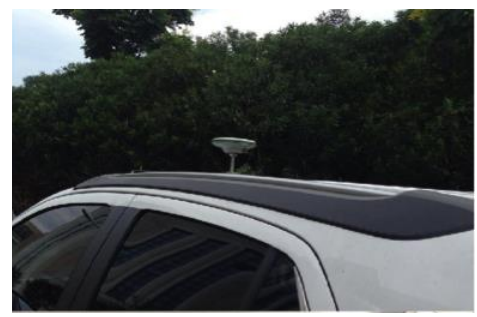

Test vehicle

Figure 3 Test equipment.

\subsection{Fusion Results with GPS/BeiDou and IMU feeds}

A prerequisite of the lane-level positioning is the knowledge of the ground truth, which can be estimated from the expensive yet accurate post-processed data from i-Mar RT-200 INS. In this section, we use GPS/BeiDou \& IMU feeds combined with advanced fusion algorithms (i.e. UPFCA/UPFCTRA) to approximate the ground truth with satisfactory accuracy.

It is expected that the multi-satellite positioning systems (with both GPS and BeiDou) will bring more redundancy of the satellites and diminish the Dilution of Precision (DOP) values, 
thereby improving the positioning accuracy. Figure 4 compares the DOP values of two systems, one based solely on GPS and the other based on both BeiDou and GPS with 15 degree elevation angle. In particular, four categories have been considered, namely the Geometric Dilution of Precision (GDOP), Position Dilution of Precision (PDOP), Horizontal Dilution of Precision (HDOP), and Vertical Dilution of Precision (VDOP). It is shown that for each category, the DOP values with BeiDou+GPS are consistently lower than those with GPS only.

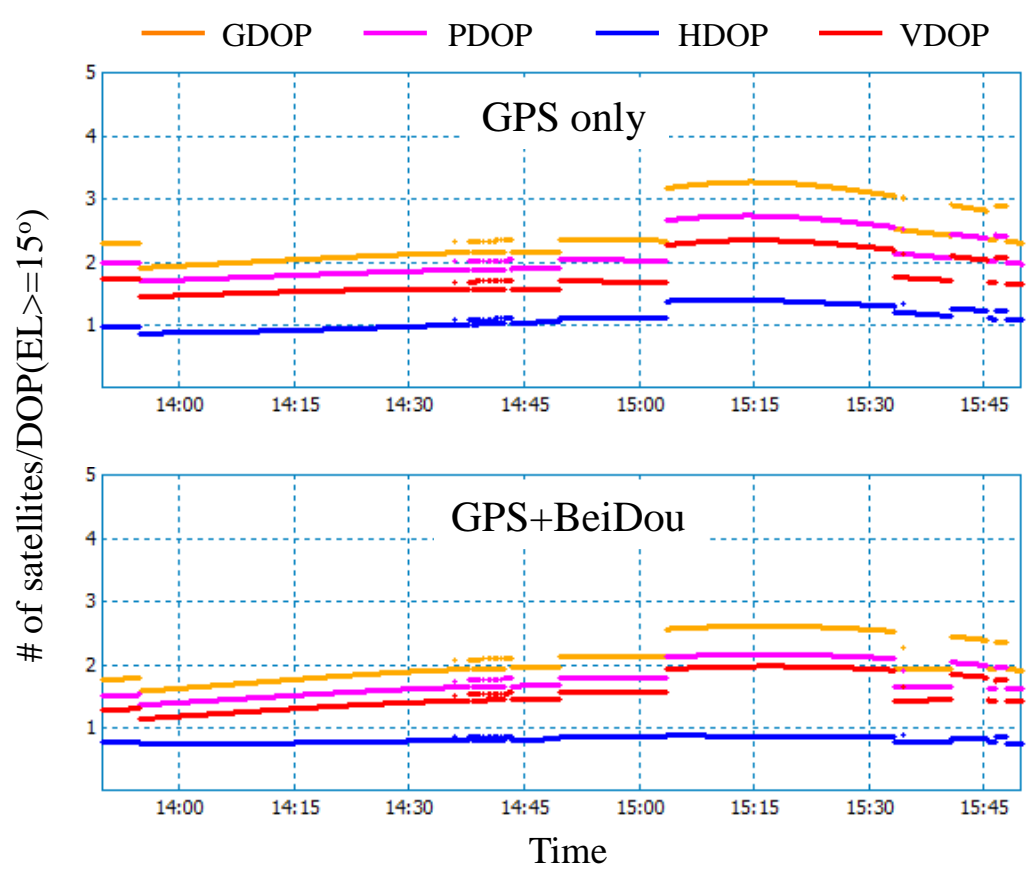

Figure 4 DOP values with GPS only and GPS+BeiDou

Table 7 shows the positioning accuracy with different GNSS sources and data fusion algorithms. It is clear that the combined GPS+BeiDou feeds have improved the positioning accuracy for all of the data fusion techniques employed. In particular, without any data fusion (indicated as 'Raw data' in the table), the percentage of measurement errors below $0.5 \mathrm{~m}$ has increased from $6.25 \%$ (with GPS only) to $8.26 \%$ (with GPS+BeiDou); and the mean error has decreased from $0.7591 \mathrm{~m}$ (with GPS only) to $0.6012 \mathrm{~m}$ (with GPS+BeiDou). In addition, the performance of both fusion methods, PFCA/PFCTRA from Sun et al. (2015) and UPFCA/UPFCTRA from this paper, have been enhanced with the introduction of the BeiDou signal. In particular, the percentages of errors below $0.5 \mathrm{~m}$ have been increased from $20.13 \%$ to $30.15 \%$ for PFCA/PFCTRA, and from $25.75 \%$ to $45.26 \%$ for UPFCA/UPFCTRA. Finally, a comparison between PFCA/PFCTRA and UPFCA/UPFCTRA shows that the latter, which is proposed in this paper, performs consistently better than the former regardless of the 
GNSS source. Figure 5 confirms that the UPFCA/UPFCTRA estimates are indeed closer to the reference (ground truth) than PFCA/PFCTRA estimates.

Table 7 Statistics of the 2dRMS positioning accuracy

\begin{tabular}{|c|c|c|c|c|c|c|}
\hline $\begin{array}{l}\text { GNSS } \\
\text { Source }\end{array}$ & Data Fusion & $\begin{array}{c}\text { Error } \\
<0.5(\mathrm{~m})\end{array}$ & $\begin{array}{c}\text { Error } \\
0.5-1(\mathrm{~m})\end{array}$ & $\begin{array}{c}\text { Error } \\
>1(\mathrm{~m})\end{array}$ & Total & $\begin{array}{c}\text { Mean } \\
\text { Error }(\mathrm{m})\end{array}$ \\
\hline \multirow{3}{*}{ GPS Only } & Raw data & $6.25 \%$ & $57.30 \%$ & $36.45 \%$ & $100 \%$ & 0.7591 \\
\hline & PFCA/PFCTRA & $20.13 \%$ & $65.27 \%$ & $14.60 \%$ & $100 \%$ & 0.5162 \\
\hline & UPFCA/UPFCTRA & $25.75 \%$ & $68.58 \%$ & $5.67 \%$ & $100 \%$ & 0.4215 \\
\hline \multirow{3}{*}{ GPS+BeiDou } & Raw data & $8.26 \%$ & $61.28 \%$ & $30.46 \%$ & $100 \%$ & 0.6012 \\
\hline & PFCA/PFCTRA & $30.15 \%$ & $54.58 \%$ & $15.27 \%$ & $100 \%$ & 0.4536 \\
\hline & UPFCA/UPFCTRA & $45.26 \%$ & $48.53 \%$ & $6.21 \%$ & $100 \%$ & 0.3821 \\
\hline
\end{tabular}

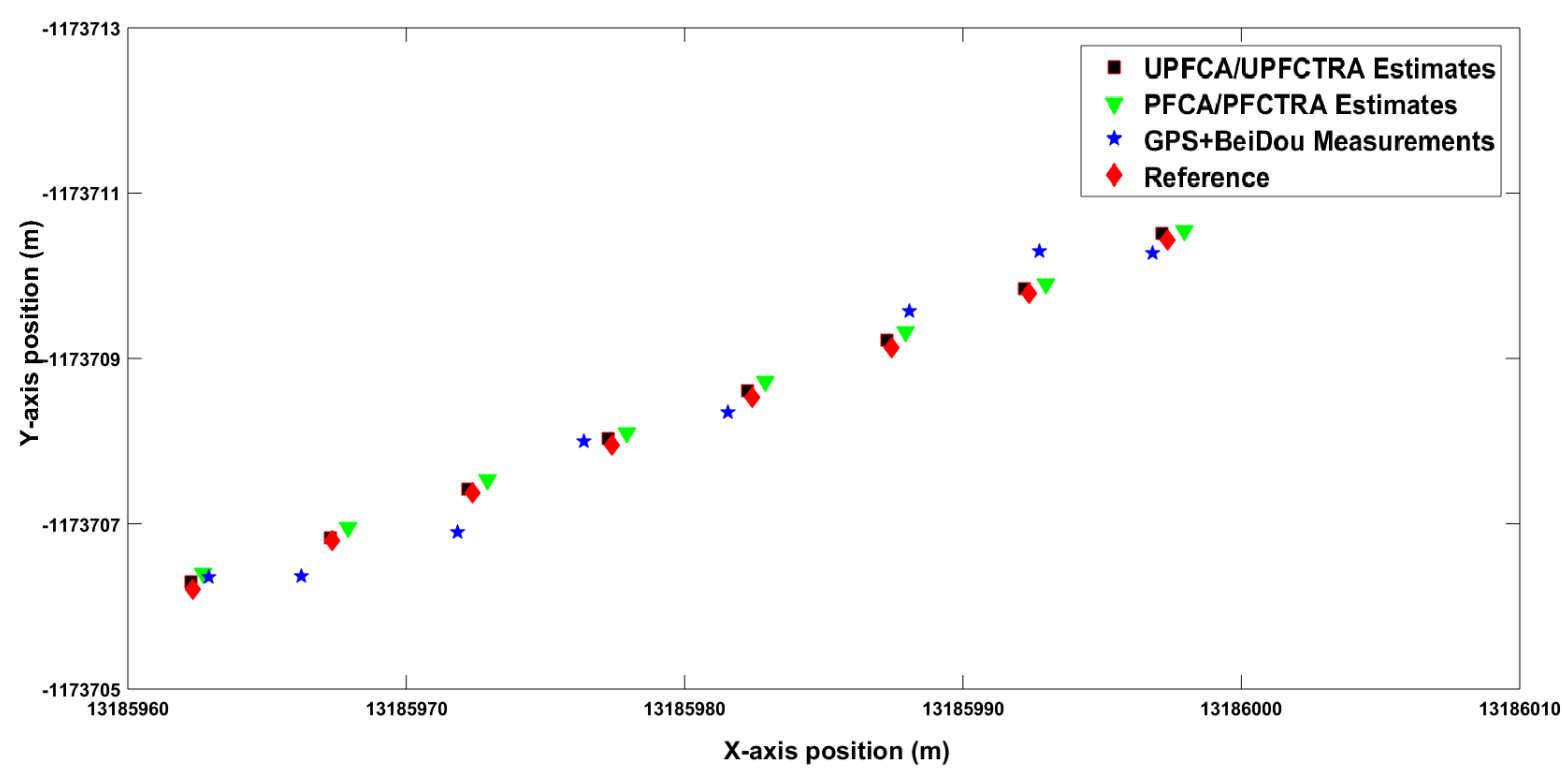

Figure 5 Comparison of positioning accuracies where the ground-truth reference is given by GPS/INS.

\subsection{Testing Anomalous Driving Detection Algorithms based on the Reference Data}

For the validation of the new FIS-based anomalous driving detection algorithm and its comparison with other state-of-the-art algorithms, we apply the reference data, which were obtained from the post-processed high grade i-Mar integrated measurements mentioned in Section 4.1. The reference data are highly accurate and thus used as the ground truth for testing the algorithms. For the defined sessions (Table 6), relevant quantities from the reference data are fed into different algorithms to produce the anomalous driving detection results in three output rates $(1 \mathrm{~Hz}, 5 \mathrm{~Hz}$ and $10 \mathrm{~Hz})$.

Here in this section, we compare the proposed algorithm with the one from our previous study (Sun et al. 2015), as well as other state-of-the-art algorithms from relevant literature. We note, according to our literature review, that no other studies have attempted to classify 
different driving styles (weaving, swerving, jerky driving, and normal driving) as we did in this paper and in Sun et al. (2015); however, some of their methodologies can still be adapted to design specific algorithms for the same application. These typical methods include Radial Basis Probability Neural Networks (RBPNN) (Chang et al., 2008), Longitude-LatitudeAcceleration based Pattern Matching Algorithm (Dai et al., 2010), and the Machine Learning algorithm designed in Krajewski et al. (2009).

The first performance measure for our comparison is time to first detection, which measures the timeliness of the detection, and the results are shown in Table 8. It can be seen that the proposed algorithm outperforms all of its competitors. In particular, the advantage of the proposed FIS-based algorithm is more pronounced with higher output rates. It is also noted from the table that, with the exception of the Pattern Matching Algorithm, the anomalous driving detection algorithms can detect anomalous driving within $5 \mathrm{~s}$ after the first incident. In addition, the Pattern Matching Algorithm produces severe delay in the detection, especially for Session 1, which can be up to $4 \mathrm{~s}$ later than our proposed algorithm. Overall, the FISbased algorithm with the V-indicator performs the best, followed by the Sun et al. (2015) algorithm and the RBPNN (Chang et al., 2000) whose performances are similar. The Machine Learning Algorithm and Pattern Matching Algorithm provide the least satisfactory results.

Table 8 Comparison of algorithms in terms of timeliness of detection (time to first detection minus time of the incident)

\begin{tabular}{|c|c|c|c|c|c|c|}
\hline \multirow{2}{*}{$\begin{array}{c}\text { Output } \\
\text { Frequency }\end{array}$} & Session 1 & Session 2 & Session 3 & Session 4 & Session 5 & Session 6 \\
\hline & \multicolumn{6}{|c|}{ Proposed FIS Algorithm with V-indi, O-indi and D-indi } \\
\hline $10 \mathrm{~Hz}$ & $1.8 \mathrm{~s}$ & $2.7 \mathrm{~s}$ & $3.1 \mathrm{~s}$ & $2.9 \mathrm{~s}$ & $2.5 \mathrm{~s}$ & $2.9 \mathrm{~s}$ \\
\hline $5 \mathrm{~Hz}$ & $2.0 \mathrm{~s}$ & $3.0 \mathrm{~s}$ & $3.5 \mathrm{~s}$ & $3.1 \mathrm{~s}$ & $2.7 \mathrm{~s}$ & $3.3 \mathrm{~s}$ \\
\hline \multirow[t]{2}{*}{$1 \mathrm{~Hz}$} & $2.0 \mathrm{~s}$ & $3.5 \mathrm{~s}$ & $4.0 \mathrm{~s}$ & $3.1 \mathrm{~s}$ & $3.2 \mathrm{~s}$ & $3.3 \mathrm{~s}$ \\
\hline & \multicolumn{6}{|c|}{ FIS Algorithm with O-indi and D-indi (Sun et al., 2015) } \\
\hline $10 \mathrm{~Hz}$ & $2.2 \mathrm{~s}$ & $3.4 \mathrm{~s}$ & $3.5 \mathrm{~s}$ & $3.5 \mathrm{~s}$ & $2.9 \mathrm{~s}$ & $3.2 \mathrm{~s}$ \\
\hline $5 \mathrm{~Hz}$ & $2.5 \mathrm{~s}$ & $3.5 \mathrm{~s}$ & $3.5 \mathrm{~s}$ & $3.6 \mathrm{~s}$ & $3.2 \mathrm{~s}$ & $3.3 \mathrm{~s}$ \\
\hline $1 \mathrm{~Hz}$ & $3.0 \mathrm{~s}$ & $3.5 \mathrm{~s}$ & $4.0 \mathrm{~s}$ & $4.1 \mathrm{~s}$ & 3.2 & $3.3 \mathrm{~s}$ \\
\hline & \multicolumn{6}{|c|}{ RBPNN Algorithm (Chang et al., 2008) } \\
\hline $10 \mathrm{~Hz}$ & $2.8 \mathrm{~s}$ & $3.2 \mathrm{~s}$ & $3.2 \mathrm{~s}$ & $3.7 \mathrm{~s}$ & $2.6 \mathrm{~s}$ & $3.2 \mathrm{~s}$ \\
\hline $5 \mathrm{~Hz}$ & $3.0 \mathrm{~s}$ & $3.5 \mathrm{~s}$ & $3.5 \mathrm{~s}$ & $4.1 \mathrm{~s}$ & $2.7 \mathrm{~s}$ & $3.3 \mathrm{~s}$ \\
\hline $1 \mathrm{~Hz}$ & $3.0 \mathrm{~s}$ & $3.5 \mathrm{~s}$ & $4.0 \mathrm{~s}$ & $4.1 \mathrm{~s}$ & $3.2 \mathrm{~s}$ & $3.3 \mathrm{~s}$ \\
\hline & \multicolumn{6}{|c|}{ Pattern Matching Algorithm (Dai et al., 2010) } \\
\hline $10 \mathrm{~Hz}$ & $5.2 \mathrm{~s}$ & $3.3 \mathrm{~s}$ & $3.4 \mathrm{~s}$ & $4.8 \mathrm{~s}$ & $3.5 \mathrm{~s}$ & $4.1 \mathrm{~s}$ \\
\hline $5 \mathrm{~Hz}$ & $5.5 \mathrm{~s}$ & $3.5 \mathrm{~s}$ & $3.5 \mathrm{~s}$ & $5.1 \mathrm{~s}$ & $3.7 \mathrm{~s}$ & $4.3 \mathrm{~s}$ \\
\hline $1 \mathrm{~Hz}$ & $6.0 \mathrm{~s}$ & $3.5 \mathrm{~s}$ & $4.0 \mathrm{~s}$ & $5.1 \mathrm{~s}$ & $4.2 \mathrm{~s}$ & $4.3 \mathrm{~s}$ \\
\hline & \multicolumn{6}{|c|}{ Machine Learning Algorithm (Krajewski et al., 2009) } \\
\hline $10 \mathrm{~Hz}$ & $3.8 \mathrm{~s}$ & $3.4 \mathrm{~s}$ & $3.4 \mathrm{~s}$ & $4.8 \mathrm{~s}$ & $3.0 \mathrm{~s}$ & $4.0 \mathrm{~s}$ \\
\hline $5 \mathrm{~Hz}$ & $4.0 \mathrm{~s}$ & $3.5 \mathrm{~s}$ & $3.5 \mathrm{~s}$ & $5.1 \mathrm{~s}$ & $3.2 \mathrm{~s}$ & $4.3 \mathrm{~s}$ \\
\hline $1 \mathrm{~Hz}$ & $4.0 \mathrm{~s}$ & $3.5 \mathrm{~s}$ & $4.0 \mathrm{~s}$ & $5.1 \mathrm{~s}$ & $3.2 \mathrm{~s}$ & $4.3 \mathrm{~s}$ \\
\hline
\end{tabular}


The second and third performance measures for our comparison are availability and correct detection rate, whose definition can be found in Section 3.3. Table 9 shows the comparison results based on the reference data. The findings are as follows.

(1) All the anomalous driving detection algorithms perform properly and yield high availability of system outputs, although our proposed method has the highest availability across all three output frequencies.

(2) In terms of the correct detection rate, the proposed algorithm outperforms the others by a discernable margin, especially compared to the algorithms (RBPNN, Pattern Matching, Machine Learning) from the literature.

(3) The Pattern Matching Algorithm yields significant error in the anomalous driving identification. A possible reason is that as the algorithm only takes, as input, the longitudinal and latitudinal acceleration, which are inadequate to capture the different driving styles considered in this paper.

Table 9 Comparison of the Algorithms in terms of availability and correct detection rate based on the reference data with different output frequencies.

\begin{tabular}{|c|c|c|}
\hline \multirow[t]{2}{*}{ Output Frequency } & Availability & Correct Detection Rate \\
\hline & \multicolumn{2}{|c|}{ Proposed FIS Algorithm with V-indi, O-indi and D-indi } \\
\hline $10 \mathrm{~Hz}$ & $88.14 \%$ & $87.12 \%$ \\
\hline $5 \mathrm{~Hz}$ & $95.18 \%$ & $95.29 \%$ \\
\hline \multirow[t]{2}{*}{$1 \mathrm{~Hz}$} & $97.05 \%$ & $97.51 \%$ \\
\hline & \multicolumn{2}{|c|}{ FIS Algorithm with O-indi and D-indi (Sun et al., 2015) } \\
\hline $10 \mathrm{~Hz}$ & $85.48 \%$ & $85.95 \%$ \\
\hline $5 \mathrm{~Hz}$ & $94.49 \%$ & $95.13 \%$ \\
\hline \multirow[t]{2}{*}{$1 \mathrm{~Hz}$} & $96.04 \%$ & $96.31 \%$ \\
\hline & \multicolumn{2}{|c|}{ RBPNN Algorithm (Chang et al., 2008) } \\
\hline $10 \mathrm{~Hz}$ & $86.28 \%$ & $78.30 \%$ \\
\hline $5 \mathrm{~Hz}$ & $93.78 \%$ & $82.82 \%$ \\
\hline \multirow[t]{2}{*}{$1 \mathrm{~Hz}$} & $95.28 \%$ & $87.93 \%$ \\
\hline & \multicolumn{2}{|c|}{ Pattern Matching Algorithm (Dai et al., 2010) } \\
\hline $10 \mathrm{~Hz}$ & $85.25 \%$ & $37.38 \%$ \\
\hline $5 \mathrm{~Hz}$ & $93.72 \%$ & $46.24 \%$ \\
\hline \multirow[t]{2}{*}{$1 \mathrm{~Hz}$} & $96.51 \%$ & $49.85 \%$ \\
\hline & \multicolumn{2}{|c|}{ Matching Learning Algorithm (Krajewski et al., 2009) } \\
\hline $10 \mathrm{~Hz}$ & $84.89 \%$ & $71.31 \%$ \\
\hline $5 \mathrm{~Hz}$ & $92.69 \%$ & $77.06 \%$ \\
\hline $1 \mathrm{~Hz}$ & $95.19 \%$ & $80.56 \%$ \\
\hline
\end{tabular}

From these results, it is clear that the anomalous driving styles (weaving, swerving, jerky driving, and normal driving) can be distinguished by the proposed algorithm along with some 
other ones from previous studies. The new FIS algorithm proposed in this paper is shown to outperform the state-of-the-arts in terms of time of first detection, availability, and correction rate.

\subsection{Integrated Approach}

In this section, the integrated and end-to-end GPS/BeiDou/IMU-fusion-detection approach for anomalous driving detection is tested and validated. Notice that this integrated approach makes contribution in all three aspects, namely data source, fusion method, and anomalous driving detection algorithm. In this experiment, the combined GPS/BeiDou signals are used as the source of the raw data, which were fed to the fusion model and then used by the proposed FIS-based anomalous driving detection algorithm. ${ }^{4}$

Based on the GPS/BeiDou/IMU data feeds, we compare the combined (fusion model $)+($ anomaly detection model). The two candidates are: UPFCA/UPFCTRA fusion model + the velocity-based FIS algorithm, as proposed in this paper; and PFCA/PFCTRA fusion model + the FIS algorithm (Sun et al., 2015). The comparison results in terms of time to first detection are presented in Table 10. It can be seen that the first combination provides more timely responses, especially with higher output rates. For example, in Session 2, the time to first detection calculated by our algorithm can be 0.9 s earlier than the Sun et al. (2015) algorithm under $10 \mathrm{~Hz}$ output frequency.

Table 10 Comparison of (data fusion model)+(irregularity detection model) in terms of time of first detection

\begin{tabular}{|c|c|c|c|c|c|c|}
\cline { 2 - 7 } \multicolumn{1}{c|}{} & \multicolumn{2}{c|}{ UPFCA/UPFCTRA + the velocity-based } & \multicolumn{2}{c|}{ PFCA/PFCTRA + the Sun et al. (2015) } \\
\hline $\begin{array}{c}\text { Output } \\
\text { Frequency }\end{array}$ & $10 \mathrm{HZ}$ & $5 \mathrm{~Hz}$ & $1 \mathrm{~Hz}$ & $10 \mathrm{~Hz}$ & $5 \mathrm{~Hz}$ & $1 \mathrm{~Hz}$ \\
\hline Session 1 & $1.9 \mathrm{~s}$ & $2.0 \mathrm{~s}$ & $2.0 \mathrm{~s}$ & $2.4 \mathrm{~s}$ & $2.5 \mathrm{~s}$ & $3.0 \mathrm{~s}$ \\
\hline Session 2 & $2.9 \mathrm{~s}$ & $3.0 \mathrm{~s}$ & $3.5 \mathrm{~s}$ & $3.8 \mathrm{~s}$ & $4.5 \mathrm{~s}$ & $4.5 \mathrm{~s}$ \\
\hline Session 3 & $3.3 \mathrm{~s}$ & $3.5 \mathrm{~s}$ & $4.0 \mathrm{~s}$ & $3.7 \mathrm{~s}$ & $4.0 \mathrm{~s}$ & $4.0 \mathrm{~s}$ \\
\hline Session 4 & $3.0 \mathrm{~s}$ & $3.1 \mathrm{~s}$ & $3.1 \mathrm{~s}$ & $3.7 \mathrm{~s}$ & $4.1 \mathrm{~s}$ & $4.1 \mathrm{~s}$ \\
\hline Session 5 & $2.6 \mathrm{~s}$ & $2.7 \mathrm{~s}$ & $3.2 \mathrm{~s}$ & $3.0 \mathrm{~s}$ & $3.2 \mathrm{~s}$ & $3.2 \mathrm{~s}$ \\
\hline Session 6 & $3.2 \mathrm{~s}$ & $3.3 \mathrm{~s}$ & $3.3 \mathrm{~s}$ & $3.5 \mathrm{~s}$ & $3.8 \mathrm{~s}$ & $4.3 \mathrm{~s}$ \\
\hline
\end{tabular}

The comparison results in terms of availability and correct detection rate are presented in Table 11. The availability and correct detection rate based on the proposed model and algorithm are higher than its competitor, especially for high-frequency outputs.

\footnotetext{
${ }^{4}$ Since the inclusion of BeiDou source is obviously beneficial to the positioning accuracy, in our comparison we have skipped the part where GPS is used as the only source of positioning.
} 
Table 11 Comparison in terms of availability and correct detection rate

\begin{tabular}{|c|c|c|c|c|}
\cline { 2 - 5 } \multicolumn{1}{c|}{} & \multicolumn{2}{c|}{ Availability } & \multicolumn{2}{c|}{ Correct Detection Rate } \\
\hline $\begin{array}{c}\text { Output } \\
\text { Frequency }\end{array}$ & $\begin{array}{c}\text { UPFCA/UPFCTRA }+ \\
\text { the velocity-based FIS } \\
\text { algorithm }\end{array}$ & $\begin{array}{c}\text { PFCA/PFCTRA }+ \\
\text { the Sun et al. (2015) } \\
\text { FIS algorithm }\end{array}$ & $\begin{array}{c}\text { UPFCA/UPFCTRA + } \\
\text { the velocity-based FIS } \\
\text { algorithm }\end{array}$ & $\begin{array}{c}\text { PFCA/PFCTRA + } \\
\text { the Sun et al. (2015) } \\
\text { FIS algorithm }\end{array}$ \\
\hline $10 \mathrm{~Hz}$ & $84.13 \%$ & $79.28 \%$ & $86.82 \%$ & $81.25 \%$ \\
\hline $5 \mathrm{~Hz}$ & $94.35 \%$ & $93.49 \%$ & $92.20 \%$ & $91.13 \%$ \\
\hline $1 \mathrm{~Hz}$ & $95.21 \%$ & $94.04 \%$ & $95.71 \%$ & $94.15 \%$ \\
\hline
\end{tabular}

It is validated that the UPFCA/UPFCTRA fusion algorithm with GPS/BeiDou source have greatly improved positioning accuracy, compared to the GPS only positioning results. Furthermore, the newly designed UPFCA/UPFCTRA model with the proposed FIS algorithm provides earlier detection than the other state-of the-art algorithms. Moreover, the availability and correct detection rate of the anomalous driving has also been improved by the same algorithm especially in higher output rates.

\section{Conclusion}

This paper presents an integrated framework for anomalous driving detection. We make contribution in three aspects: data source, fusion model, and anomalous driving identification algorithm. We are among the first to introduce BeiDou as an additional source of positioning for anomalous driving detection, and have demonstrated its effectiveness and practicality in terms of deployment and financial cost. Then, we propose two new data fusion models, Unscented Kalman Filter and Unscented Particle Filter, to estimate vehicle motion parameters. Last but not least, a FIS-based algorithm that takes into account the velocity indicator is proposed. The advantages of individual components have been demonstrated using extensive tests involving both simulated and real-world data.

The integrated anomalous driving detection system not only utilizes BeiDou feeds, which significantly improves vehicle positioning results, but also relies on a series of methodological advancements during various stages of the process with much improved timeliness, availability, and correctness of the detection. The field test has demonstrated the practicality of this approach for real-world applications through the use of low-cost equipment and integrated procedures.

\section{Acknowledgements}

The authors are grateful to Dr. Xu Tang and Mr. Yiming Quan from the University of Nottingham, Ningbo Campus for their help with the field experiment. The contribution of $\mathrm{Mr}$. 
Bin Wang from the Zhoushan Police Station in China is also acknowledged for providing the traffic data. This research work is supported by "the Fundamental Research Funds for the Central Universities", NO. NJ20160015.

\section{References}

Cai, C., Gao, Y., Pan L., 2014. An analysis on combined GPS/COMPASS data quality and its effect on single point positioning accuracy under different observing conditions. Advances in Space Research 54, 818-829.

Chang, T. H., Hsu, C. S., Wang, C., Yang, L. K., 2008. Onboard measurement and warning module for anomalous vehicle behavior, IEEE Transactions of. Intelligent Transportation Systems 9 (3), 501-513.

Chen, H-C., Huang, Y-S., Chiang, K-W., Yang, M., Rau, R-J., 2009. The performance comparison between GPS and BeiDou - 2/ compass: A perspective from Asia. Journal of the Chinese Institute of Engineers 32 (5): 679-689.

Dai, J., Teng, J., Bai, X., Shen, Z., Xuan, D., 2010. Mobile phone based drunk driving detection. 4th International Conference on Computing Technologies for Healthcare (Pervasive Health), 1.

Gyorgy, K., Kelemen, A., David, L., 2014. Unscented Kalman filters and Particle Filter methods for nonlinear state estimation. Procedia Technology 12, 65-74.

Imkamon, T., Saensom, P., Tangamchit, P., Pongpaibool, P., 2008. Detection of hazardous driving behavior using fuzzy logic, 5th International Conference on Electrical Engineering/Electronics, Computer, Telecommunications and Information Technology. ECTI-CON 2008, 2, 657. 
Krajewski, J., Sommer, D., Trutschel, U., Edwards, D., Golz, M., 2009. Steering wheel behavior based estimation of fatigue, Proceedings of the Fifth International Driving Symposium on Human Factors in Driver Assessment, Training and Vehicle Design, 118-124.

Lecce, V.D., Calabrese, M., 2008. Experimental system to support real time driving pattern recognition, Advanced Intelligent Computing Theories and Applications with Aspects of Artificial Intelligence Annals of Emergency Medicine, 1192-1199.

NHTSA. (2010). http://www.nhtsa.gov/

Odolinski, R., Teunissen, P. J. G., Odijk, D., 2014. First combined COMPASS/BeiDou-2 and GPS positioning results in Australia. Part I: single-receiver and relative code-only positioning. Journal of Spatial Science 59 (1), 3-24.

Odolinski, R., Teunissen, P. J. G., Odijk, D., 2014. First combined COMPASS/BeiDou-2 and GPS positioning results in Australia. Part II: Single-and multiple frequency single-baseline RTK positioning. Journal of Spatial Science 59 (1), 25-46.

Saruwatari, K., Sakaue, F., Sato, J., 2012. Detection of abnormal driving using multiple view geometry in space-time, IEEE Intelligent Vehicles Symposium (IV), 1102-1107.

Sun, F., Liu, S., 2012. Research and progress of BeiDou satellite navigation system. Science China 55 (12), 2899-2907.

Sun, R., Ochieng, W. Y., Feng, S, 2015. An integrated solution for lane level irregular driving detection on highways. Transportation Research Part C 56, 61-79.

Tsogas, M., Polychronopoulos, A., Amditis, A., 2005. Unscented Kalman filter design for curvilinear motion models suitable for automotive safety applications, IEEE Proceedings of 
7th International Conference on Information Fusion, 1295-1302.

Van Der Merwe, R., Wan E., 2000. The square-root unscented Kalman filter for state and parameter estimation, Oregon Graduate Institute of Science and Technology, Oregon. Available:http://www.researchgate.net/publication/2895916 The_Square_Root_Unscented_K al man_Filter_for_State_and_Parameter-Estimtion.

Van der Merwe, R., Doucet, A., de Freitas, J . F. G., Wan, E., 2000. The unscented particle filter, Technical Report CUED/F-INFENG/TR 380, Cambridge University Engineering Department.

Wan, E., Van Der Merwe, R., 2000. The unscented Kalman filter for nonlinear estimation. Adaptive Systems for Signal Processing, Communications, and Control Symposium. ASSPCC. The IEEE 2000, 153-158.

Xiao, G., Sui, L., Liu, C., Lu, Z., Gan, Y., Qi, G., 2014. A method of determining the weight matrix for BeiDou Navigation System single point positioning. Acta Geodaetica et Cartographica Sinica 43 (9), 902-907.

Xu, J., Yang, Y., Li, J., He, H., Guo, H., 2013. Integrity analysis of COMPASS and other GNSS combined navigation. Science China: Earth Sciences 56, 1616-1622.

Yang, Y., 2010. Progress, contribution and challenges of Compass/BeiDou satellite navigation system. Acta Geodaetica et Cartographica Sinica (In Chinese) 39 (1), 1-6. 